\title{
Next generation sequencing for profiling expression of miRNAs: technical progress and applications in drug development
}

\author{
Jie Liu' ${ }^{1}$, Steven F. Jennings ${ }^{2}$, Weida Tong ${ }^{3}$, Huixiao Hong ${ }^{3 *}$ \\ ${ }^{1}$ University of Arkansas at Little Rock/University of Arkansas for Medical Sciences Bioinformatics Graduate Program, Little Rock, \\ USA; \\ ${ }^{2}$ Department of Information Science, University of Arkansas at Little Rock, Little Rock, USA; \\ ${ }^{3}$ National Center for Toxicological Research, US Food and Drug Administration, Jefferson, USA. \\ Email: ${ }^{*}$ Huixiao.Hong@fda.hhs.gov
}

Received 28 July 2011; revised 1 September 2011; accepted 19 September 2011.

\begin{abstract}
miRNAs are non-coding RNAs that play a regulatory role in expression of genes and are associated with diseases. Quantitatively measuring expression levels of miRNAs can help in understanding the mechanisms of human diseases and discovering new drug targets. There are three major methods that have been used to measure the expression levels of miRNAs: realtime reverse transcription PCR (qRT-PCR), microarray, and the newly introduced next-generation sequencing (NGS). NGS is not only suitable for profiling of known miRNAs as qRT-PCR and microarray can do too but it also is able to detect unknown miRNAs which the other two methods are incapable of doing. Profiling of miRNAs by NGS has progressed rapidly and is a promising field for applications in drug development. This paper reviews the technical advancement of NGS for profiling miRNAs, including comparative analyses between different platforms and software packages for analyzing NGS data. Examples and future perspectives of applications of NGS profiling miRNAs in drug development will be discussed.
\end{abstract}

Keywords: miRNAs; Next-Generation Sequencing; Expression; Data Analysis; Drug Development

\section{INTRODUCTION}

miRNAs (also known as microRNAs) are endogenous, non-coding ribonucleic acids (RNAs) approximately twenty-one nucleotides in length. First encountered in 1993 at Harvard University [1], a rapid increase in published articles referring to miRNA ensued as shown in Figure 1. A testament to the significant and increasing scientific interest that is driven by research needs and fostered by rapid advances in technologies for measuring expression levels of miRNAs, it is now clear that miRNAs play a fundamental role in normal tissue development. With an important regulatory role in expression of genes, they are associated with diseases involving multiple changes in gene expression, including cancer and viral infections. Therefore, quantitatively measuring expression levels of miRNAs facilitates the understanding of mechanisms of human diseases and the discovering of new drug targets.

Primarily, there are three methods that are used to measure the expression levels of miRNAs: real-time reverse transcription PCR (qRT-PCR), microarray hybridization, and, more recently, next-generation sequencing (NGS). NGS not only profiles known miRNAs as do the more traditional methods (e.g., qRT-PCR and microarrays), but it is also able to identify unknown miRNAs which are beyond the capabilities of these traditional methods. Profiling miRNAs by NGS has progressed rapidly

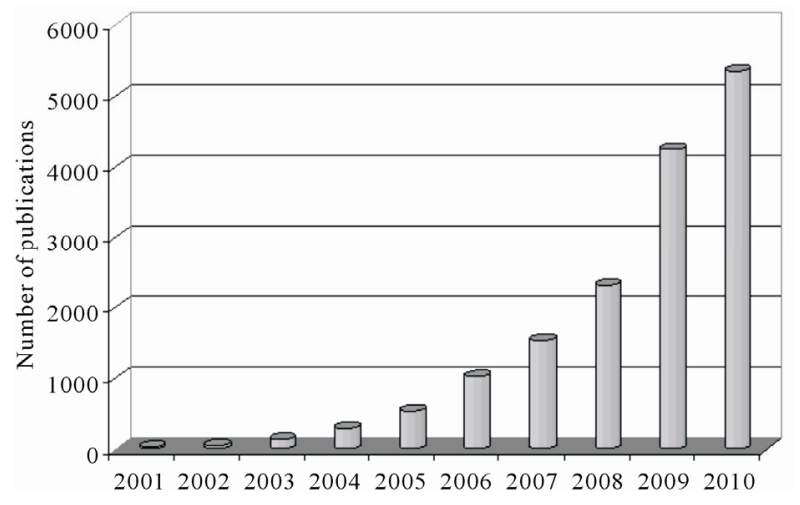

Figure 1. Annual number of publications related to miRNAs from 2001 to 2010 based on a keyword search in PubMed. Keyword used: microRNA. (Search was conducted on Jul. 11, 2011). 
and is a promising field for applications in drug development. This paper will summarize the technical advancement of NGS for profiling miRNAs, including comparative analyses between different platforms, experimental protocols, algorithms for matching short reads to known miRNA sequences, strategies for quantitatively measuring expression levels, and methods for detecting unknown miRNAs. Different pipelines and software packages for analyzing NGS data for profiling of miRNAs will be reviewed. Examples and future perspectives of applications of NGS profiling miRNAs in drug development will be discussed.

\section{BIOLOGY OF miRNAs}

miRNAs are on average only twenty-one nucleotides long, expressed from longer transcripts encoded in animal, plant and virus genomes. miRNAs are transcribed as long precursors, pri-miRNAs, and then cleaved to $\sim 65 \mathrm{nt}$ hairpin-shaped, precursor pre-miRNAs by the RNase III enzyme Drosha and its cofactor DGCR8 (DiGeorge syndrome critical region gene 8 or Pasha) (Figure 2). Pre-miRNAs are further processed to generate mature miRNAs. miRNAs are post-transcriptional regulators that bind to complementary sequences on target messenger RNA transcripts (mRNAs), usually resulting in translational repression and gene silencing $[2,3]$.

The first miRNA was discovered in 1993 during a study of the gene lin-14 in C. elegans development [1]. However, miRNAs were not recognized as a distinct class of biologic regulators with conserved functions until the early 2000's when a second miRNA (let-7) was characterized. Since then, researches have revealed multiple roles of miRNAs in negative regulation (e.g., transcript degradation and sequestering and translational suppression) and possible involvement in positive regulation (e.g., transcriptional and translational activation). Currently, there are over 16,000 miRNAs from over one hundred species in the miRNA registry, miRBase [4], including about 1400 human miRNAs [5].

miRNAs repress their target genes' expression by recognition of the eight nucleotides (seed sequences) on the 3' UTR of the genes [6]. As such, the relationship between miRNAs and their target genes are many-to-many: a single miRNA may target multiple genes and a single gene may contain recognition sites for multiple miRNAs. Most human genes are the conserved targets of miRNAs [7]. miRNAs are involved in all major biological process, including the regulation of most physiological processes: cell proliferation [8], cell apoptosis [9,10], metabolism [11], and development and morphogenesis [12,13].

The breadth and importance of miRNA-directed gene regulation are coming into focus as more miRNAs and their regulatory targets and functions are discovered.

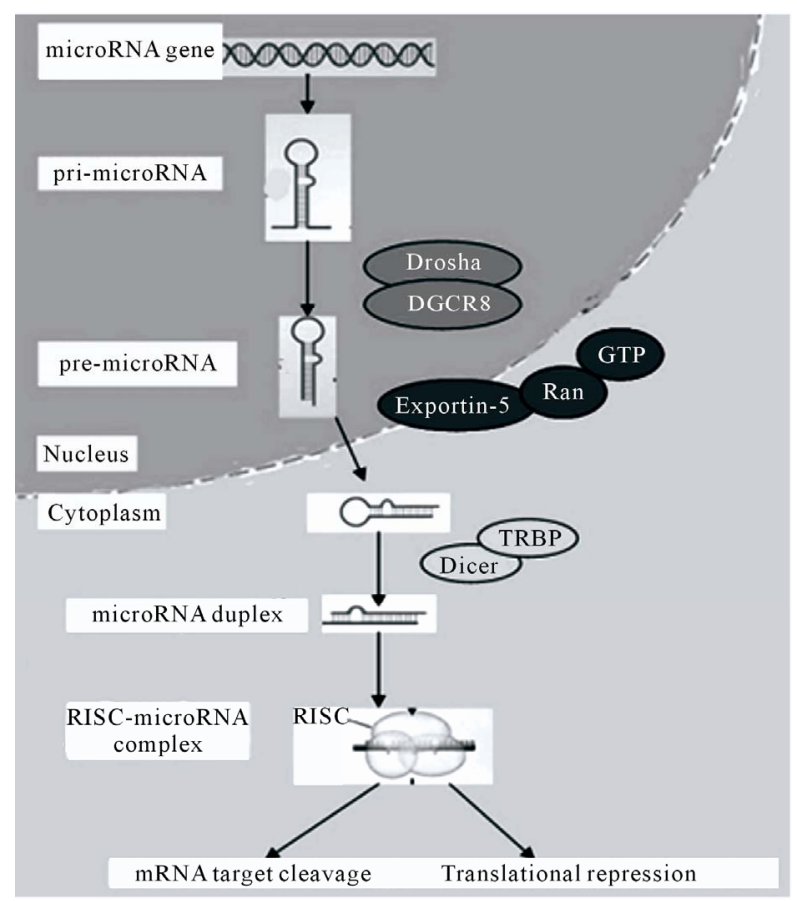

Figure 2. miRNA biogenesis and action.

Given the ability of miRNAs to target multiple genes and key biological processes, these molecules have received intensive research interest both as biomarkers and as therapeutic agents [14-16].

miRNAs appear to be involved in many diseases [17], including diabetes [18], cardiomyopathies [19], psychiatric disorders including schizophrenia [20,21], and cancer [22]. In cancer, miRNAs may exert oncogenic functions by inhibiting tumor suppressor genes or may act as tumor suppressors by inhibiting oncogenes [23,24].

\section{EXPRESSION OF MiRNAs}

The key question for miRNA research is which miRNAs are active under a given set of experimental conditions and how does that pattern change in the dynamic cellular environment. The technical challenge is to precisely measure miRNA expression levels. Northern blotting was the earliest technique that attempted to systematically profile miRNA expression in various experimental systems [25]. The primary methods currently used for measuring the expression levels of miRNAs are qRT-PCR [26,27], microarray hybridization [28,29], and next generation sequencing [30].

qRT-PCR is a laboratory technique used to generate multiple copies of a DNA sequence by using a pair of primers that are complementary to the sequence on each of the two strands of the cDNA. It consists of three major steps: reverse transcription (RT), denaturation, and DNA extension. RT transcribes RNA to cDNA using reverse transcriptase. The denaturation step then sepa- 
Table 1. Some popular miRNA microarray platforms.

\begin{tabular}{|c|c|c|c|}
\hline Company & Platform & miRBase & Species \\
\hline Agilent & $\begin{array}{l}\text { Human miRNA } \\
\text { Microarray } 8 \times 60 \mathrm{~K}\end{array}$ & $16.0(2010)$ & Human \\
\hline Agilent & $\begin{array}{l}\text { Mouse miRNA } \\
\text { Microarray 8x60K }\end{array}$ & $16.0(2010)$ & Mouse \\
\hline Agilent & $\begin{array}{l}\text { Rat miRNA Microarray } \\
8 \times 15 \mathrm{~K}\end{array}$ & $16.0(2010)$ & Rat \\
\hline Affymetrix & $\begin{array}{c}\text { GeneChip }^{\circledR} \text { miRNA } 2.0 \\
\text { Array }\end{array}$ & $15.0(2010)$ & multiple \\
\hline Illumina & $\begin{array}{l}\text { MicroRNA Expression } \\
\text { Profiling Assay }\end{array}$ & $12.0(2008)$ & multiple \\
\hline Exiqon & $\begin{array}{l}\text { miRCURY LNA }{ }^{\mathrm{TM}} \\
\text { microRNA Array }\end{array}$ & $16.0(2010)$ & multiple \\
\hline $\begin{array}{l}\text { Life Tech- } \\
\text { nologies }\end{array}$ & $\begin{array}{l}\text { NCode }^{\mathrm{TM}} \text { Human } \\
\text { miRNA Microarray V3 }\end{array}$ & $10.0(2007)$ & Human \\
\hline
\end{tabular}

rates the strands and the primers can bind again at lower temperatures and begin a new chain reaction. Finally, DNA extension from the primers is done with thermostable Taq DNA polymerase. qRT-PCR is a sensitive method for measuring expression levels of precursor or mature miRNAs. It requires low amounts of starting material. With the design of RT primers with high specificity towards individual mature miRNA species, multiple primers could be potentially combined in a single pool that would enable much higher throughput profiling than is currently possible with individual sample analyses. qRT-PCR is suitable for quantification of a few known miRNAs in a large number of samples.

miRNA microarray hybridization is a popular method for measuring miRNA expression levels because a large number of miRNAs can be measured simultaneously [29]. Several companies offer miRNA microarray platforms which are designed and developed based on the same miRNAs database, miRBase [4]. Therefore, the major difference among the miRNA microarray platforms is the miRNAs on the arrays. Because this field is evolving very rapidly, users should select a platform based on the version of miRBase which contains the most relevant information for their samples. Companies frequently update their contents to reflect a newer miRBase release. Table 1 lists some popular miRNA microarray platforms.

\section{NGS}

Rapid determination of DNA sequence base pairs, first reported by Sanger [35,36], provided a tool to decipher genes. However, low throughput and-more importantly-high cost hindered using this sequencing technology for deciphering the human genome. A breakthrough came in 2005 when the sequencing-by-synthesis technology developed by 454 Life Sciences was published [37]. Since then, several NGS platforms, such as Illumina Genome Analyzer (Illumina, Inc., San Diego, CA, USA) and SOLiD ${ }^{\mathrm{TM}}$ (Life Technologies Corporation, Carlsbad, CA, USA), have been developed and applied to various fields of biological and medical research, including measuring expression levels of known miRNAs and detecting unknown miRNAs.

\subsection{NGS Platforms}

Different NGS platforms use divergent sequencing chemistries. Table 2 compares the main features of three NGS platforms, but it is important to note that these values are constantly changing as newer models are released. Both Illumina Genome Analyzer system and SOLiD use short-read sequencing technologies. The Roche 454 Genome Sequencer has the advantage of longer sequence reads and is the best choice for denovo sequencing of new genomes.

\subsubsection{Illumina Genome Analyzer}

The Illumina Genome Analyzer system currently is the most widely-used, short-read sequencing platform. It applies the sequencing-by-synthesis method. miRNAs are first reversely translated to DNA and the DNA samples are randomly sheared into fragments, then ligated to oligonucleotide adapters at both ends. Single-stranded DNA fragments are attached to reaction chambers and are extended and amplified by bridge PCR amplification with fluorescently-labeled nucleotides for sequencing. The Genome Analyzer is widely used by many genome sequencing projects for its consistent data quality and proper read lengths. The new Illumina HiSeq 2000 has dramatically increased throughput.

\subsubsection{Roche 454 Genome Sequencer FLX}

The 454 Genome Sequencer uses the principle of pyro-

Table 2. Comparison of NGS platforms.

\begin{tabular}{|c|c|c|c|}
\hline & \multicolumn{3}{|c|}{ Platform } \\
\hline & Illumina & Roche 454 & SOLiD \\
\hline & $\begin{array}{l}\text { Sequencing- } \\
\text { by-synthesis }\end{array}$ & Pyrosequencing & $\begin{array}{l}\text { Ligation-based } \\
\text { sequencing }\end{array}$ \\
\hline Applification & Bridge PCR & Emulsion PCR & Emulsion PCR \\
\hline Read length & $35-150 b p$ & $\sim 400 \mathrm{bp}$ & $75 / 35$ bp \\
\hline $\begin{array}{l}\text { Paired ends/ } \\
\text { separation }\end{array}$ & Yes/200 bp & Yes/3000 bp & Yes/3000 bp \\
\hline $\mathrm{Mb} /$ run & $1300 \mathrm{Mb}$ & $100 \mathrm{Mb}$ & $3000 \mathrm{Mb}$ \\
\hline $\begin{array}{c}\text { Time/run } \\
\text { (paired ends) }\end{array}$ & 4 days & 7 hours & 5 days \\
\hline Comments & $\begin{array}{c}\text { Most widely } \\
\text { used }\end{array}$ & $\begin{array}{l}\text { Longer reads, fast } \\
\text { run, higher cost }\end{array}$ & $\begin{array}{l}\text { Good data } \\
\text { quality }\end{array}$ \\
\hline
\end{tabular}


sequencing. Sheared DNA fragments are ligated to specific oligonucleotide adapters and are amplified by emulsion PCR on the surfaces of agarose beads. The current maximum read length of the 454 platform is $600 \mathrm{bp}$ which is the longest short-read among all of the NGS platforms. Thus, the 454 Genome Sequencer FLX is best suited for applications requiring longer reads, such as RNA isoform identification in RNA-seq and de novo assembly of microbes in metagenomics [38].

\subsubsection{Applied Biosystems SOLiD Sequencer}

The Applied Biosystems SOLiD sequencer uses the sequencing-by-ligation approach and may offer the best data quality. It amplifies sheared DNA fragments by an emulsion PCR approach with small magnetic beads. But the DNA library preparation procedures prior to sequencing currently take five days which is both tedious and time consuming.

\subsection{Sequence Alignment}

A NGS experiment generates huge amount of sequence data. Consequently, there is a high demand for bioinformatics tools to cope with these large amounts of sequencing data. The key process in NGS data analysis is to align the huge amount of short reads to a given genome. A variety of algorithms and software packages have been specifically developed for dealing with millions of NGS short-read alignments (Table 3).

Bowtie (http://bowtie.cbcb.umd.edu/) is an ultrafast and efficient alignment program for aligning short sequences to large genomes [39]. Bowtie indexes the reference genome using a scheme based on the BurrowsWheeler index to keep its memory footprint small. It does not use an exact matching algorithm, which is quite common in other tools, because exact matching does not directly allow for sequencing errors or genetic variations. The two key algorithmic strategies that make Bowtie extremely fast in alignment of short reads are backtracking and double indexing. Backtracking allows mismatches and favors high-quality alignments, while the double in-

Table 3. Short-read sequence alignment tools.

\begin{tabular}{ll}
\hline Name & Description \\
Bowtie & $\begin{array}{l}\text { Uses a Burrows-Wheeler transform to create a per- } \\
\text { manent, reusable index of the genome; faster run for } \\
\text { short sequence alignment to reference genome }\end{array}$ \\
BWA & $\begin{array}{l}\text { Slower than bowtie but allows indels in alignment } \\
\text { performs only ungapped alignments and allows up to } \\
\text { three mismatches }\end{array}$ \\
SeqMap & $\begin{array}{l}\text { Up to } 5 \text { mixed substitutions and insertions/deletions. } \\
\text { Various tuning options and input/output formats. }\end{array}$ \\
SOAP & $\begin{array}{l}\text { Allow up to } 3 \text { gaps and mismatches. SOAP2 uses } \\
\text { bidirectional BWT to build the index of reference and } \\
\text { increases the running speed. }\end{array}$ \\
TopHat & Splice junction mapper for RNA-Seq reads \\
\hline
\end{tabular}

dexing avoids excessive backtracking.

$B W A$ (http://maq.sourceforge.net/) is a software package for aligning short sequencing reads against a large reference sequence such as the human genome [40]. This alignment algorithm is based on a backward search with the Burrows-Wheeler Transform (BWT) of the reference genome. It allows mismatches and gaps for single-end reads. BWA also supports paired-end mapping. It generates a mapping quality index and gives multiple hits if requested.

MAQ (http://maq.sourceforge.net/) is a software package for rapidly mapping shotgun short reads to a reference genome and using quality scores to derive genotype calls of the consensus sequence of a diploid genome [41]. MAQ searches for the ungapped match with lowest mismatch score. For each alignment, a quality score is assigned to measure the probability that the true alignment is not the one found by $M A Q$. Using $M A Q$, users can map reads, call consensus sequences including single nucleotide polymorphisms (SNPs) and indel variants, simulate diploid genomes and read sequences, and postprocess the results in various ways.

SeqMap (http://www.stanford.edu/group/wonglab/jiangh/ seqmap/) is a tool for aligning a large number of short sequences to a reference genome [42]. It explores the whole reference genome for each short read se- quence from NGS data. Multiple substitutions and insertions/deletions are allowed in SeqMap. It accepts FASTA input format and output results in various formats. Parallel computing with SeqMap on a cluster of computers is supported. This program is fast, usually taking just a few hours on a desktop PC for a typical alignment of NGS data.

SOAP (http://soap.genomics.org.cn/) is a program for efficient gapped and ungapped alignment of short read sequences onto reference sequences [43]. It adopts a seed-and-hash look-up table algorithm to accelerate its alignment process. First, short reads and the reference sequences are converted to a numeric data type using a 2-bits-per-base encoding system. Then the look-up table is checked to determine how many bases are different between a short read and a reference sequence. SOAP is a command-driven program and supports multi-threaded, parallel computing. It accepts FASTA format for reference and both FASTA and FASTQ formats for input short reads. With $S O A P$, users can do single-read or pairend resequencing, small RNA discovery, and mRNA tag sequence mapping.

TopHat (http://tophat.cbcb.umd.edu/) is designed to align RNA-Seq reads and to create a view of the junctions, or to align to a known set of junctions [44]. The TopHat pipeline consists of multiple steps. Initially, all the short reads are aligned to the reference genome 
using Bowtie. The short reads that have not been aligned to the genome are set aside as "initially unmapped reads". The aligned short reads are then assembled to generate possible splices between neighboring exons sequences flanking potential donor/acceptor splice sites with neighboring exons joined together to generate potential splice junctions. Then, the "initially unmapped reads" are aligned with these potential splice junction sequences.

\subsection{NGS for Profiling miRNAs}

\subsection{1. miRNA Databases}

NGS short reads are aligned to a known reference sequence database. These miRNA databases are the repository for known miRNA sequence and annotation data. They are the core of NGS data analysis for expression profiling of miRNAs. The most important and popular miRNA databases include, but not limited to: miRBase [4], deepBase [45], microRNA.org [46], miRGen 2.0 [47], miRNAMap [48], and PMRD [49].

The miRBase database (http://www.mirbase.org) is an online database repository for known miRNAs. It provides an integrated web interface to analyze miRNA sequence data and to predict gene targets. The miRBase database has three main functions: 1) the miRBase Registry acts as an independent arbiter of miRNA gene nomenclature, assigning names prior to publication of novel miRNA sequences; 2) the miRBase Sequences provides known miRNA sequence data, references, and links to other resources; and 3) the miRBase Targets is used for the prediction of miRNA target genes.

The deepBase (http://deepbase.sysu.edu.cn/) is a comprehensive web-based database for annotating and discovering small and long non-coding RNAs, ncRNAs (miRNAs, siRNAs, piRNAs, etc.), from high-throughput deep sequencing data. In the current version of deepBase, NGS data from 185 small RNA libraries from diverse tissues and cell lines of seven organisms (human, mouse, chicken, Ciona intestinalis, Drosophila melanogaster, Caenhorhabditis elegans, and Arabidopsis thaliana) have been curated. Its integrative, interactive, and versatile web graphical interface facilitates analyzing and visualizing NGS data on the internet.

The microRNA.org (http://www.microrna.org/ microrna/ home.do) is a database for experimentally-observed miRNA expression patterns and predicted miRNA targets and target downregulation scores. Through its graphical interface, the microRNA.org web resource provides several functions: 1) exploring genes that are potentially regulated by a particular miRNA; 2) searching for the set of miRNAs that potentially regulate a particular gene cooperatively; and 3) comparing miRNA expression profiles in different tissues. The strategy for miRNA target prediction is to treat miRNAs as adaptors of genes in the 3'-UTR region by using near-prefect, base-pairing in a small region in the 5 ' end (positions 2-8) of the miRNA. The most valuable information of this web resource is the experimental data that can be used to verify miRNA regulation.

The miRGen 2.0 (http://diana.cslab.ece.ntua.gr/ mirgen/) is a database of miRNA genomic information and regulation. It contains 812 human miRNA coding transcripts and 386 mouse miRNA coding transcripts as well as expression profiles of 548 human and 451 mouse miRNAs and over 172 human and 68 mouse small RNA libraries derived from cell lines and tissues. It is implemented in a MySQL relational database management system. Its interface allows users to search for miRNAs and transcription factors of interest.

The miRNAMap (http://mirnamap.mbc.nctu.edu.tw/) provides genomic maps of miRNAs and their target genes in mammalian genomes. Experimentally-verified miRNAs and experimentally-verified miRNA target genes in human, mouse, rat, and other metazoan genomes are collected in this database. Target genes of miRNAs are predicted using three bioinformatics tools: miRanda [50], TargetScan [51], and RNAhybrid [52] independently. To reduce false positives for predicted target genes, three strategies were used: 1) a predicted target site is one that is predicted by at least two of the three bioinformatics tools; 2) the target site must be within an accessible region of a gene; and 3) the target gene has to have multiple target sites. Its interface is well designed to facilitate access to the data and analyzing data as well as visualizing the data and associated analysis results.

PMRD (http://bioinformatics.cau.edu.cn/PMRD/) is a plant miRNA database. This database contains 8433 miRNAs from 121 plant species and possible target genes for each miRNA with a predicted interaction site. The data in PMRD include available plant miRNA data deposited in the public database, the ones curated from literature, and miRNA profiling data generated in-house.

\subsection{2. miRNA NGS Data Analysis Tools}

There are several web servers and standalone programs for analysis of miRNA expression profiling and novel miRNA discovery from NGS data. The web servers include miRanalyzer [53,54] and miRCat [55]. Examples of standalone analysis tools are miRDeep [56] and miRExpress [57].

miRanalyzer (http://web.bioinformatics.cicbiogune.es/ microRNA/) is a web server for identifying and analyzing miRNA in deep-sequencing data. After inputting NGS data (a list of unique reads and their copy numbers) into the web server tool, users can conduct data analysis in three steps: 1) detect all known miRNA sequences 
annotated in miRBase; 2) match against other libraries of transcribed sequences; and 3) predict new miRNAs. It accepts two different input file formats: 1) a tab-separated file with a row representing a short read sequence and its count and 2) a multi-FASTA format file with the copy number of the unique short reads (read count) as the description in the header. To detect the expression levels of known miRNAs - a major objective of many miRNAs studies - miRanalyzer aligns the short reads to the known miRNA sequences using the miRBase repository [4] which offers mature (the mature sequences of known miRNAs), mature-star (the sequence which pairs with the mature miRNA in the pre-miRNA secondary structure), and precursor miRNA sequences (sequence of the hairpin). Since a group of miRNAs that can be aligned with the same read normally belong to the same family, miRanalyzer reports these ambiguous matches, stating all miRNAs where alignments were found. After known miRNAs are detected, their corresponding target genes (the genes predicted to be regulated by the detected miRNA) are predicted and precalculated ontological analyses are given. For the remaining reads that are not aligned to the known miRNAs, miRanalyzer maps them to databases of transcribed sequences as miRNA, noncoding RNA, and (retro)-transposons. The mapping step is "perfect", meaning that no mismatch is allowed in the mapping. In the last step - the most important task of miRNA NGS data analysis - detecting previously-unreported miRNAs is conducted. A machine-learning approach based on the random forest method [58] is used to detect new miRNAs in miRanalyzer.

The miRCat (http://srna-tools.cmp.uea.ac.uk/) is a miRNA NGS data analysis tool that was developed for identification of mature miRNAs and their precursors. It takes a FASTA format file of small RNA reads as input and then maps them to a reference sequence database using PatMaN [59]. Analysis results from miRCat are output in three files: 1) a comma-separated text file with the details for predicted miRNA candidates; 2) the RNAfold output for candidate precursors; and 3) a FASTA format file of predicted mature miRNA sequences. The miRCat program has been tested on several high-throughput plant sRNA datasets and showed high sensitivity and specificity.

The miRDeep package (http://www.mdc-berlin.de/en/ research/research_teams/systems_biology_of_gene_regu latory_elements/projects/miRDeep/) is a stand-alone tool for identifying known and novel miRNAs from NGS data. The miRDeep program employs a probabilistic model of miRNA biogenesis to score the compatibility of the nucleotide position and frequency of sequenced RNA reads with the secondary structure of the miRNA precursor. The false positive rate and the sensitivity of its predictions are statistically controlled in miRDeep. Therefore, not only known and novel miRNAs can be detected from deep-sequencing data by using miRDeep, but also the quality of the detection results can be estimated. The key function of miRDeep is the detection of miRNAs by analyzing how sequenced RNAs are compatible with the way miRNA precursors are processed in the cell. It should be noted is that miRDeep is designed to detect miRNAs without cross-species comparisons.

The miRExpress (http://miRExpress.mbc.nctu.edu. tw) software package is written in the $\mathrm{C}++$ programming language and can be executed on 32- or 64-bit Linux machines. It was developed to extract miRNA expression profiles from sequencing reads obtained by NGS technology. The data analysis pipeline of miRExpress consists of three steps. In the first step, the identical short reads are merged into a unique read with "a count of reads." Each unique short read is also checked to deter- mine whether it contains a full or a partial adaptor sequence. In the second step, each unique short read is aligned with the sequences of known mature miRNAs in miRBase [4]. In the third step, expression levels of miRNAs are measured by computing the sum of read counts for each miRNA according to the alignment criteria (e.g., the length of the read equals the length of the miRNA sequence and the identity of the alignment is $100 \%$ ). The cutoff of alignment identity can be set by users based on their requirements when using miRExpress.

\section{APPLICATIONS OF NGS IN DRUG DEVELOPMENT}

Drug development is a complex and lengthy process (Figure 3) that starts from pharmaceutical target identification and validation followed by lead identification and optimization (usually termed as drug discovery). The lead compounds are subsequently subjected to preclinical tests and clinical trials of increasing levels of complexity (usually termed as drug development). Following the completion of all three phases of clinical trials, a pharmaceutical company analyzes all of the data and files a new drug application with the FDA. If the data on the new drug successfully demonstrate both safety and effectiveness in careful review, the FDA approves the new drug to be distributed on the market. For some drugs, the FDA requires additional trials (commonly called Phase IV) to evaluate long-term effects. This whole process (including discovery and development) for a new drug takes about ten to five years and costs around $\$ 1$ - $\$ 2$ billion [60,61].

In a relatively short time, high-throughput sequencing of small RNAs have provided a great potential for the profiling of known and novel small RNAs. Given the 


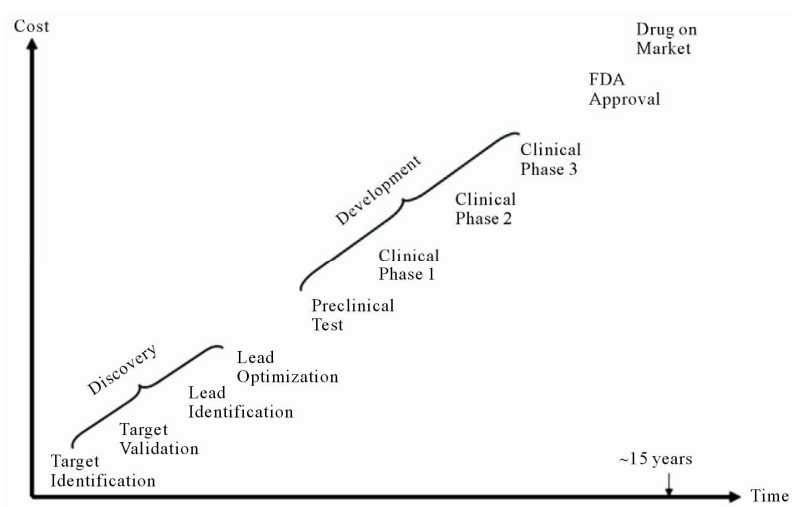

Figure 3. Drug development process.

ability of miRNAs to target multiple genes and play a major role in most biological processes, miRNAs have received intensive research interest both as biomarkers and as therapeutic agent targets $[9,15,23,62]$ for drug development.

Some miRNAs are involved in the regulation of tumorigenesis and are tumor tissue-specific. Therefore, these miRNAs are potential biomarkers for diagnosis and prognosis of cancers and could serve as pharmaceutical targets for development of anti-cancer drugs [16]. For example, twenty-two miRNAs were up-regulated and thirteen were down-regulated in gastric cancer [63]. In that study, $m i R-125 b, m i R-199 a$, and $m i R-100$ were also found to be involved in the progression of gastric cancer. Another example is the let-7 miRNA. It regulates the RAS oncogene. Expression of let-7 miRNA in some human lung tumors causes increased expression of the RAS oncogene and may contribute to tumorigenesis [24]. Expression levels of miRNAs have been shown to play a controlling role in tumor growth rates, suggesting possible new strategies for therapeutic treatment [64]. The serum levels of miR-141 (a miRNA expressed in prostate cancer) differ significantly between prostate cancer patients and healthy controls [65].

Some miRNAs are particularly abundant in the nervous system and regulate processes such as neurogenesis, synapse development, and plasticity in the brain, controlling the expression of hundreds of genes involved in neuroplasticity and synapses. For example, it was observed that the expression level of miR-16 causes adaptive changes in production of the serotonin trans- porter, miR-133b regulates the production of tyrosine hydroxylase and the dopamine transporter, and miR-212 affects production of striatal brain-derived neurotrophic factor and synaptic plasticity upon cocaine $[14,62]$. These miRNAs could be potential drug targets for more efficient therapies.

A recent study found that the miRNA ( $m i R-101)$ target sites within Alzheimer's amyloid- $\beta$ precursor protein
(APP) 3'-untranslated region (3'-UTR) and down-regulates APP levels in human cell cultures. It is differentially expressed and involved in the regulatory network of Alzheimer's disease (AD). The results suggest that $m i R-101$ could be a potential target for $\mathrm{AD}$ therapeutics [66].

Recently, NGS has been applied to identify miRNA biomarkers for diagnosis and prognosis of disease. For example, the expression levels of the oncogenic miRNAs of the miR17-92 cluster and the miR-181 family determined by using SOLiD NGS were higher in five unfavorable neuroblastomas. In contrast, the expression levels of the tumor suppressive miRNAs of miR-542-5p and $m i R-628$ were much higher in five favorable neuroblastomas compared to the five unfavorable neuroblastomas in which the expressions of these two miRNAs were virtually absent [14].

Theoretically, inhibition of a particular miRNA involved in a disease can block the expression of a therapeutic target protein and administration of a miRNA mimetic can boost the endogenous miRNA population repressing a detrimental gene. Therefore, miRNA inhibitors and some miRNA mimetics can serve as therapeutic candidates for various diseases such as cancer, cardiovascular disease, neurological disorders, and viral infection. A lot of pharmaceutical companies are invested in the development of drugs that target miRNAs. Currently, a number of new drug products targeting miRNAs are in pre-clinical studies and in clinical trials. Table 4 summarizes some of these. A detailed example of the utilization of miRNAs in drug development will be reviewed.

A liver specific miRNA, $m i R-122$, regulates a host of messenger RNAs in the liver, many of which encode proteins involved in lipid and cholesterol metabolism. It is abundant in healthy individuals. Replication of hepatitis $\mathrm{C}$ virus (HCV) is dependent on $m i R-122$ expression [67]. When normal liver cells are infected by $\mathrm{HCV}, \mathrm{miR}-122$ binds to the two target sites located in the 5 '-end of the HCV genome, increasing infectious virus production [68] as depicted in Figure 4 (box A). Therefore, if a drug can be designed to specifically recognize and bind to miR-122, the replication process of HCV will be effectively blocked as shown in Figure 4 (box B). Miravirsen (SPC-3649), developed by Santaris Pharma (http://www.santaris.com/), is such an inhibitor of $m i R-122$ and is expected to provide a very high barrier to the generation of viral resistance. Pre-clinical studies showed a potent, dose-dependent, and long lasting inhibition of miR-122 in mice, cynomologus monkeys, and green African monkeys as indicated by decreases in cholesterol levels. A phase II clinical study in patients infected with HCV started in September 2010 and cur- 
Table 4. Examples of miRNAs in drug development.

\begin{tabular}{ccccc}
\hline Generic Name & Target & Indication & Status & Company \\
\hline Miravirsen & miR-122 & Hepatitis C virus & Phase IIA & Santaris Pharma \\
Unspecified & miR-21 & Fibrosis & Clinical & Regulus Therapeutics \\
Unspecified & miR-21 & Cancer & Clinical & Regulus Therapeutics \\
Unspecified & mi-R122 & Hepatitis C virus & Clinical & Regulus Therapeutics \\
Unspecified & mi-155 & Inflammation & Pre-clinical & Regulus Therapeutics \\
Unspecified & miR-33a & Metabolic diseases & Pre-clinical & Regulus Therapeutics \\
MGN-9103 & miR-208/499 & Chronic Heart Failure & Pre-clinical & Miragen Therapeutics \\
MGN-1374 & miR-15/195 & Post-MI Remodeling & Pre-clinical & Miragen Therapeutics \\
MGN-4893 & miR-451 & Polycythemia Vera & Pre-clinical & Miragen Therapeutics \\
MGN-4420 & miR-29 & Cardiac fibrosis & Lead optimization & Miragen Therapeutics \\
Unspecified & Let-7 & Lung cancer & Pre-clinical & Mirna Therapeutics \\
Unspecified & miR-34 & Prostate cancer & Pre-clinical & Mirna Therapeutics \\
TCDD & miR-191 & Hepatocellular carcinoma & Pre-clinical & Rosetta Genomics \\
Unspecified & miR-34a & Liver cancer & Pre-clinical & Rosetta Genomics \\
\hline
\end{tabular}

rently SPC-3649 is in a multiple ascending dose study in healthy volunteers. SPC-3649 is the first drug targetting miRNA to enter human clinical trials.

\section{FUTURE PERSPECTIVE}

Overall, manipulation of miRNA functions through the activation or the silencing of miRNAs could become a promising therapeutic tool and novel strategy for disease treatment. NGS technologies have facilitated an enormous potential for miRNA detection and gene regulation research for drug development. miRNA expression profiling plays an important role in the identification of new therapeutic strategies, clinical diagnostics, and personalized medicine $[23,69,70]$.

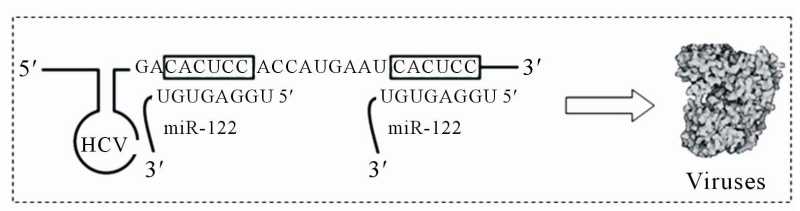

(a)

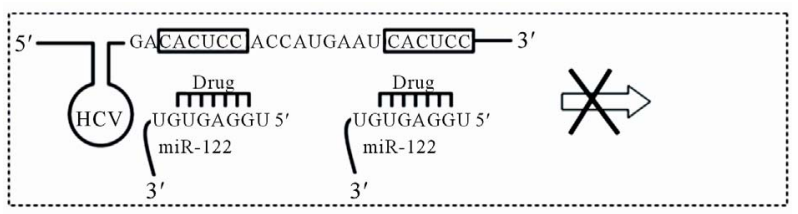

(b)

Figure 4. Schematic view of the role of miR-122 in HCV replication (a) and binding of a drug to miR-122 to block HCV replication (b).
The roles of miRNAs in diseases have been very well established over the last few years. Although there is still much to be learned concerning the mechanism of miRNAs in biological processes, scientists have been able to apply their knowledge to use miRNAs as biomarkers for diagnosis and prognosis of diseases and as potential pharmaceutical targets for drug development. Within the last few years, many studies on miRNAs have moved into animal models with highly encouraging results for drug development. With the progress of NGS technology, expression levels of known miRNAs will be more precisely and rapidly detected and more and more novel miRNAs will be discovered as biomarkers for diagnosis and prognosis of diseases and as potential targets for drug development. Thus, we look forward to a brighter future for utilizing miRNA expression profiling in the drug development process.

\section{ACKNOWLEDGEMENTS}

This publication was made possible by NIH Grant \# P20 RR-16460 from the IDeA Networks of Biomedical Research Excellence (INBRE) Program of the National Center for Research Resources. The views presented in this article are those of the authors and do not necessarily reflect those of the US Food and Drug Administration. No official endorsement is intended nor should be inferred.

\section{REFERENCES}

[1] Lee, R.C., Feinbaum, R.L. and Ambros, V. (1993) The C. elegans heterochronic gene lin-4 encodes small RNAs with antisense complementarity to lin-14. Cell, 75, 843854. doi:10.1016/0092-8674(93)90529-Y 
[2] Bartel, D.P. (2004) MicroRNAs: Genomics, biogenesis, mechanism, and function. Cell, 116, 281-297. doi:10.1016/S0092-8674(04)00045-5

[3] Bartel, D.P. (2009) MicroRNAs: Target recognition and regulatory functions. Cell, 136, 215-233. doi:10.1016/i.cell.2009.01.002

[4] Griffiths-Jones, S., Saini, H.K., van Dongen, S. and Enright, A.J. (2008) miRBase: Tools for microRNA genomics. Nucleic Acids Research, 36, D154-D158.

[5] Sheng, Y., Engstrom, P.G. and Lenhard, B. (2007) Mammalian microRNA prediction through a support vector machine model of sequence and structure. PLoS One, 2, e946. doi:10.1371/journal.pone.0000946

[6] van den Berg, A., Mols, J. and Han, J. (2008) RISCtarget interaction: Cleavage and translational suppression. Biochimica Biophysica Acta, 1779, 668-677.

[7] Friedman, R.C., Farh, K.K., Burge, C.B. and Bartel, D.P. (2009) Most mammalian mRNAs are conserved targets of microRNAs. Genome Research, 19, 92-105.

[8] Bueno, M.J., de Castro, I.P. and Malumbres, M. (2008) Control of cell proliferation pathways by microRNAs. Cell Cycle, 7, 3143-3148. doi:10.1101/gr.082701.108

[9] He, L. and Hannon, G.J. (2004) MicroRNAs: small RNAs with a big role in gene regulation. Nature Reviews $\mathrm{Ge}$ netics, 5, 522-531. doi:10.1038/nrg1379

[10] Jovanovic, M. and Hengartner, M.O. (2006) microRNAs and apoptosis: RNAs to die for. Oncogene, 25, 61766187. doi:10.1038/sj.onc. 1209912

[11] Krutzfeldt, L. and Stoffel, M. (2006) MicroRNAs: A new class of regulatory genes affecting metabolism. Cell Matabolism, 4, 9-12. doi:10.1016/j.cmet.2006.05.009

[12] Stefani, G. and Slack, F.J. (2008) Small noncoding RNAs in animal development. Nature Reviews Molar Cell Biology, 9, 219-230. doi:10.1038/nrm2347

[13] He, X., Eberhart, J.K. and Postlethwait, J.H. (2009) MicroRNAs and micro-managing the skeleton in diease, development, and evolution. Journal of Cellular and Molecular Medicine, 13, 606-618. doi:10.1111/j.1582-4934.2009.00696.x

[14] Schulte, J.H., Marschall. T., Martin, M., et al. (2010) Deep sequencing reveals differential expression of microRNAs in favorable versus unfavorable neuroblastoma. Nucleic Acids Research, 38, 5919-5928. doi:10.1093/nar/gkq342

[15] Fasanaro, P., Greco, S., Ivan, M., Capogrossi, M.C. and Martelli, F. (2010) microRNA: Emerging therapeutic targets in acute ischemic diseases. Pharmacology Therapeutics, 125, 92-104. doi:10.1016/j.pharmthera.2009.10.003

[16] Trang, P., Weidhaas, J.B. and Slack, F.J. (2008) MicroRNAs as potential cancer therapeutics. Oncogene, 27, S52-S57.doi:10.1038/onc.2009.353

[17] Jiang, Q., Wang, Y., Hao, Y., et al. (2009) miR2Disease: A manually curated database for microRNA deregulation in human disease. Nucleic Acid Research, 37, D98-D104. doi:10.1093/nar/gkn714

[18] Hennessy, E. and O'Driscoll, L. (2008) Molecular medicine of microRNAs: Structure, function, and implications for diabetes. Expert Reviews in Molecular Medicine, 10, e24. doi:10.1017/S1462399408000781

[19] van Rooij, E., Sutherland, L.B., Liu, N., et al. (2006) A signature pattern of stress-responsive microRNAs that can evoke cardiac hypertrophy and heart failure. Proceedings of the National Academy of Sciences, 103, 18255-18260. doi:10.1073/pnas.0608791103

[20] Barbato, C., Giorge, C., Catalanotto, C. and Cogoni C. (2008) Thinking about RNA? MicroRNAs in the brain. Mammalian Genome, 19, 541-551. doi:10.1007/s00335-008-9129-6

[21] Beveridge, N.J., Gardiner, E., Carroll, A.P., et al. (2009) Schizophrenia is associated with an increase in cortical microRNA biogenesis. Molecular Psychiatry, 15, 11761189. doi:10.1038/mp.2009.84

[22] Medina, P.P. and Slack, F.J. (2008) microRNAs and cancer: An overview. Cell Cycle, 7, 2485-2492. doi:10.4161/cc.7.16.6453

[23] Nana-Sinkam, S.P. and Croce, C.M. (2011) MicroRNAs as therapeutic targets in cancer. Translational Research, 157, 216-225. doi:10.1016/j.trsl.2011.01.013

[24] Chen, C.Z. (2005) MicroRNAs as oncogenes and tumor suppressors. The New England Journal of Medicine, 353, 1768-1771. doi:10.1056/NEJMp058190

[25] Lagos-Quintana, M., Rauhut, R., Lendeckel, W. and Tuschl, T. (2001) Identification of novel genes coding for small expressed RNAs. Science, 294, 853-858. doi:10.1126/science.1064921

[26] Chen, C., Ridzon, D.A., Broomer, A.J., et al. (2005) Realtime quantification of microRNAs by stem-loop RT-PCR. Nucleic Acids Research, 33, e179. doi:10.1093/nar/gni178

[27] Shi, R. and Chiang, V.L. (2005) Facile means for quantifying microRNA expression by real-time PCR. Biotechniques, 39, 519-525. doi:10.2144/000112010

[28] Yin, J.Q., Zhao, R.C. and Morris, K.V. (2008) Profiling microRNA expression with microarrays. Trends Biotechnol, 26, 70-76. doi:10.1016/j.tibtech.2007.11.007

[29] Li, W. and Ruan, K. (2009) MicroRNA detection by microarray. Analytical Bioanalytical Chemistry, 394, 11171124. doi:10.1007/s00216-008-2570-2

[30] Hafner, M., Landgraf, P., Ludwig, J., et al. (2008) Identification of microRNAs and other small regulatory RNAs using cDNA library sequencing. Methods, 44, 3-12. doi:10.1016/j.ymeth.2007.09.009

[31] Roush, S. and Slack, F.J. (2008) The let-7 family of microRNAs. Trends in Cell Biology, 18, 505-516. doi:10.1016/j.tcb.2008.07.007

[32] Buermans, H.P., Ariyurek, Y., van Ommen, G., et al. (2010) New methods for next generation sequencing based microRNA expression profiling. BMC Genomics, 11, 716. doi:10.1186/1471-2164-11-716

[33] Metzker, M.L. (2010) Sequencing technologies-the next generation. Nature Reviews Genetics, 11, 31-46. doi: $10.1038 / \mathrm{nrg} 2626$

[34] 't Hoen, P.A, Ariyurek, Y., Thygesen, H.H., et al. (2008) Deep sequencing-based expression analysis shows major advances in robustness, resolution and inter-lab portability over five microarray platforms. Nucleic Acids Research, 36, e141.doi:10.1093/nar/gkn705

[35] Sanger, G., Air, G.M., Barrell, B.G., et al. (1977) Nucleotide sequence of bacteriophage X174 D. Nature, 265, 687695.

[36] Sanger, F., Nicklen, S., Coulson, A.R., et al. (1977) DNA sequencing with chain-terminating inhibitors. Proceedings of the National Academy of Sciences, 74, 5463- 
5467. doi:10.1073/pnas.74.12.5463

[37] Margulies, M., Egholm, M., Altman, W.E., et al. (2005) Genome sequencing in microfabricated high-density picolitre reactors. Nature, 437, 376-380.

[38] Mocali, S. and Benedetti, A. (2010) Exploring research frontiers in microbiology: The challenge of metagenomics in soil microbiology. Research in Microbiology, 161, 497-505. doi:10.1016/j.resmic.2010.04.010

[39] Langmead, B., Trapnell, C., Pop, M. and Salzberg, S.L. (2009) Ultrafast and memory-efficient alignment of short DNA sequences to the human genome. Genome Biology, 10, R25. doi:10.1186/gb-2009-10-3-r25

[40] Li, H. and Durbin, R. (2009) Fast and accurate short read alignment with Burrows-Wheeler transform. Bioinformatics, 25, 1754-1760. doi:10.1093/bioinformatics/btp324

[41] Li, H.; Ruan, J. and Durbin, R. (2008) Mapping short DNA sequencing reads and calling variants using mapping quality scores. Genome Research, 18, 1851-1858. doi:10.1101/gr.078212.108

[42] Jiang, H. and Wong, W.H. (2008) SeqMap: Mapping massive amount of oligonucleotides to the genome. Bioinformatics, 24, 2395-2396. doi:10.1093/bioinformatics/btn429

[43] Li, R., Li, Y., Kristiansen, K. and Wang, J. (2008) SOAP: short oligonucleotide alignment program. Bioinformatics, 24, 713-714. doi:10.1093/bioinformatics/btn025

[44] Trapnell, C., Pachter, L. and Salzberg, S.L. (2009) TopHat: discovering splice junctions with RNA-Seq. Bioinformatics, 25, 1105-1111. doi:10.1093/bioinformatics/btp120

[45] Yang, J.H., Shao, P., Zhou, H., Chen, Y.Q. and Qu, L.H. (2010) deepBase: A database for deeply annotating and mining deep sequencing data. Nucleic Acids Research, 38, D123-D130. doi:10.1093/nar/gkp943

[46] Betel, D., Wilson, M., Gabow, A., Marks, D.S. and Sander, C. (2008) The microRNA.org resource: Targets and expression. Nucleic Acids Research, 36, D149-D153. doi:10.1093/nar/gkm995

[47] Alexiou, P., Vergoulis, T., Gleditzsch, M., et al. (2010) miRGen 2.0: A database of microRNA genomic information and regulation. Nucleic Acids Research, 38, D137D141. doi:10.1093/nar/gkp888

[48] Hsu, S.D., Chu, C.H. and Tsou, A.P. (2008) miRNAMap 2.0: Genomic maps of microRNAs in metazoan genomes. Nucleic Acids Research, 36, D165-D169. doi:10.1093/nar/gkm1012

[49] Zhang, Z., Yu, J., Li, D., et al. (2010) PMRD: Plant microRNA database. Nucleic Acids Research, 38, D806D813. doi:10.1093/nar/gkp818

[50] John, B., Enright, A.J., Aravin, A., et al. (2004) Human MicroRNA targets. PLoS Biology, 2, e363. doi:10.1371/journal.pbio.0020363

[51] Lewis, B.P., Shih, I.H., Jones-Rhoades, M.W., et al. (2003) Prediction of mammalian microRNA targets. Cell, 115, 787-798. doi:10.1016/S0092-8674(03)01018-3

[52] Kruger, J. and Rehmsmeier, M. (2006) RNAhybrid: microRNA target prediction easy, fast and flexible. Nucleic Acids Research, 34, W451-W454. doi:10.1093/nar/gk1243

[53] Hackenberg, M., Sturm, M., Langenberger, D., FalcónPérez, J.M. and Aransay, A.M. (2009) miRanalyzer: A
microRNA detection and analysis tool for next-generation sequencing experiments. Nucleic Acids Research, 37, W68-W76.

[54] Hackenberg, M., Rodríguez-Ezpeleta, N. and Aransay, A.M. (2011) miRanalyzer: An update on the detection and analysis of microRNAs in high-throughput sequencing experiments. Nucleic Acids Research, 39, W132W138. doi:10.1093/nar/gkr247

[55] Moxon, S., Schwach, F. and Dalmay, T. (2008) A toolkit for analysing large-scale plant small RNA datasets. Bioinformatics, 24, 2252-2253. doi:10.1093/bioinformatics/btn428

[56] Friedländer, M.R., Chen, W., Adamidi, C., et al. (2008) Discovering microRNAs from deep sequencing data using miRDeep. Nature Biotechnology, 26, 407-415. doi:10.1038/nbt1394

[57] Wang, W.C., Lin, F.M. and Chang, W.C. (2009) miRExpress: Analyzing high-throughput sequencing data for profiling microRNA expression. BMC Bioinformatics, 10, 328. doi:10.1186/1471-2105-10-328

[58] Breiman, L. (2001) Random forests. Machine Learning, 45, 5-32. doi:10.1023/A:1010933404324

[59] Prüfer, K., et al. (2008) PatMaN: Rapid alignment of short sequences to large databases. Bioinformatics, 24, 1530-1531. doi:10.1093/bioinformatics/btn223

[60] DiMasi, J.A., Hansen, R.W. and Grabowski, H.G. (2003) The price of innovation: New estimates of drug development costs. Journal of Health Economics, 22, 151-185. doi:10.1016/S0167-6296(02)00126-1

[61] Adams, C.P. and Brantner, V.V. (2006) Estimating the cost of new drug development: Is it really $\$ 802$ million? Health Affairs, 25, 420-428. doi:10.1377/hlthaff.25.2.420

[62] Dreyer, J.L. (2010) New insights into the roles of microRNAs in drug addiction and neuroplasticity. Genome Medicine, 2, 92. doi:10.1186/gm213

[63] Ueda, T., Volinia, S, Okumura, H., et al. (2010) Relation between microRNA expression and progression and prognosis of gastric cancer: A microRNA expression analysis. Lancet Oncol, 11, 136-146. doi:10.1016/S1470-2045(09)70343-2

[64] Kumar, M.S., Lu, J., Mercer, K.L., et al. (2007) Impaired microRNA processing enhances cellular transformation and tumorigenesis. Nature Genetics, 39, 673-677. doi:10.1038/ng2003

[65] Mitchell, P.S., Parkin, R.K., Kroh, E.M., et al. (2008) Circulating microRNAs as stable blood-based markers for cancer detection. Proceedings of the National Academy of Sciences, 105, 10513-10518. doi:10.1073/pnas.0804549105

[66] Long, J.M. and Lahiri, D.K. (2011) MicroRNA-101 downregulates Alzheimer's amyloid- $\beta$ precursor protein levels in human cell cultures and is differentially expressed. Biochem and Biophysical Research Communations, 404, 889-895. doi:10.1016/j.bbrc.2010.12.053

[67] Jopling, C.L., Yi, M., Lancaster, A.M., et al. (2005) Modulation of hepatitis $\mathrm{C}$ virus RNA abundance by a liver-specific MicroRNA. Science, 309, 1577-1581. doi:10.1126/science.1113329

[68] Villanueva, R.A., Jangra, R.K., Yi, M., et al. (2010) miR122 does not modulate the elongation phase of hepatitis $\mathrm{C}$ virus RNA synthesis in isolated replicase complexes. Antiviral Research, 88, 119-123. 
doi:10.1016/i.antiviral.2010.07.004

[69] Su, Z., Ning, B., Fang, H., et al. (2011) Next-generation sequencing and its applications in molecular diagnostics. Expert Review of Molecular Diagnonstics, 11, 333-343.
[70] Rogers, G.B. and Bruce, K.D. (2010) Next-generation sequencing in the analysis of human microbiota:essential considerations for clinical application. Molecular Diagnosis \& Therapy, 14, 343-350. 